\title{
Exploiting Rational Assembly to Map Distinct Roles of Regulatory Cues during Autoimmune Therapy
}

Robert S. Oakes, ${ }^{1,2}$ Lisa H. Tostanoski, ${ }^{1}$ Senta M. Kapnick, ${ }^{1}$ Eugene Froimchuk, ${ }^{1}$ Sheneil K. Black, ${ }^{1}$ Xiangbin Zeng, ${ }^{1}$ Christopher M. Jewell1,2,3,4,5,*

${ }^{1}$ Fischell Department of Bioengineering, University of Maryland, 3102 A. James Clark Hall, 8278 Paint Branch Drive, College Park, MD, 20742, USA

2 United States Department of Veterans Affairs, VA Maryland Health Care System, 10 N Greene St, Baltimore, MD, 21201, USA

${ }^{3}$ Robert E. Fischell Institute for Biomedical Devices, 5102 A. James Clark Hall, 8278 Paint Branch Drive, College Park, MD, 20742, USA

${ }^{4}$ Department of Microbiology and Immunology, University of Maryland Medical School, 685 West Baltimore Street, HSF-I Suite 380, Baltimore, MD, 21201, USA

${ }^{5}$ Marlene and Stewart Greenebaum Comprehensive Cancer Center, 22 S Greene St, Baltimore, MD, 21201, USA

\section{SUPPORTING INFORMATION}

Fig. S1. Dose-dependent control of inflammatory pathways.

Fig. S2. Dose-dependent suppression of paralysis in EAE model of MS.

Fig. S3. Charge approximations for components, and surface charge and size of immune complexes.

Fig. S4. GpG in complexes suppresses inflammatory gene expression.

Fig. S5. GpG in complexes inhibits upstream Myd88 gene expression.

Fig. S6. GpG in complexes did not impact Ccl22 and Il4 gene expression.

Fig. S7. Dose matching to isolate the role of each component.

Fig. S8. Treatments require myelin self-antigen to suppress autoimmune driven paralysis.

Fig. S9. GpG restrains $\mathrm{CpG}$ agonist function in IFN regulatory genes in vitro. 

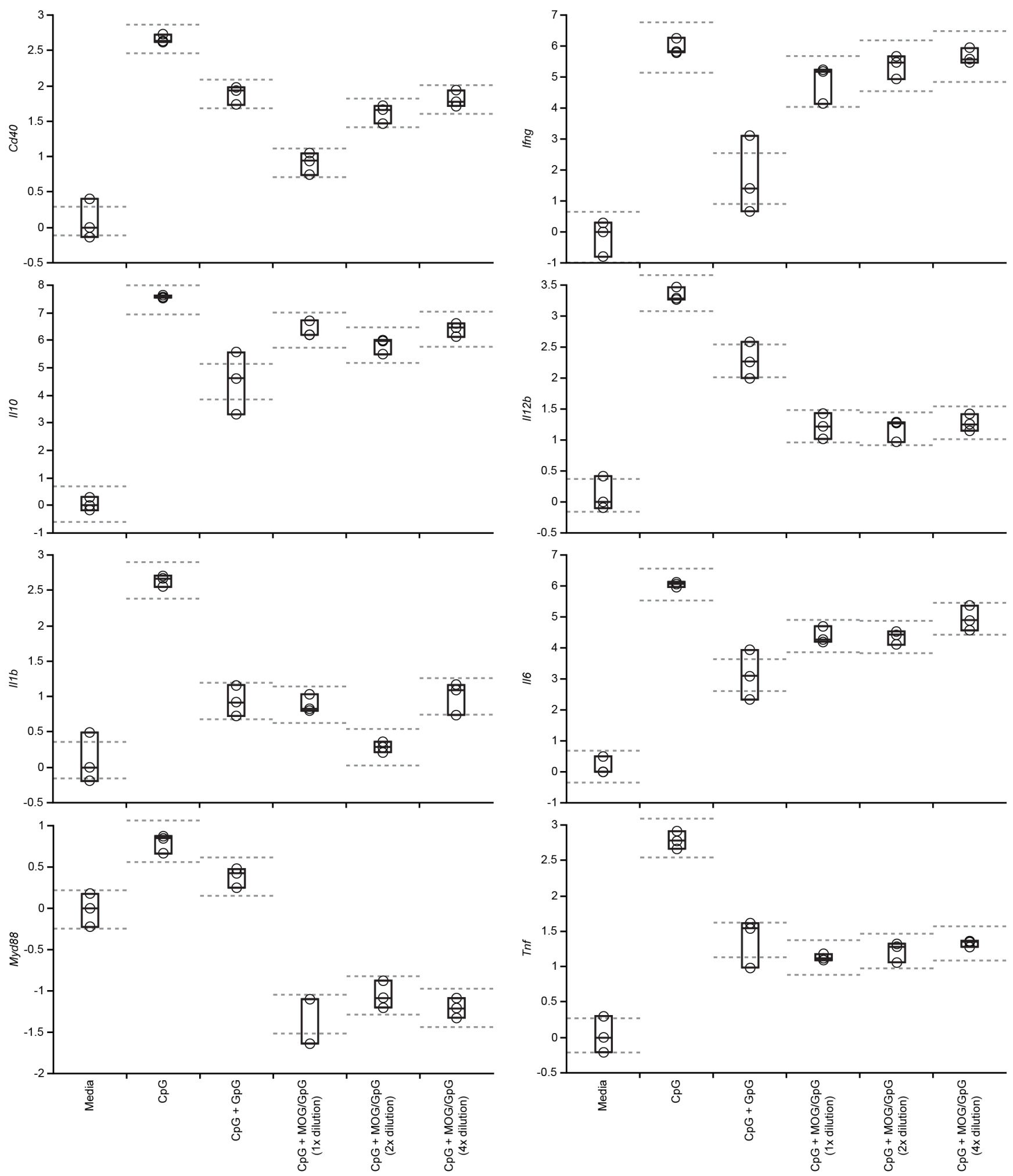

Fig. S1. Dose-dependent control of inflammatory pathways. Gene expression in DCs isolated from spleens of C57BL/6 mice were analyzed following stimulation with $\mathrm{CpG}$ and treatment with MOG/GpG complexes at 2-fold dilutions then compared against media only, CpG only, or $\mathrm{CpG}$ and soluble GpG. Box plots correspond to the heatmap in Fig. 3E. Data is centered on media groups with y-axis indicated the log2 fold change. 


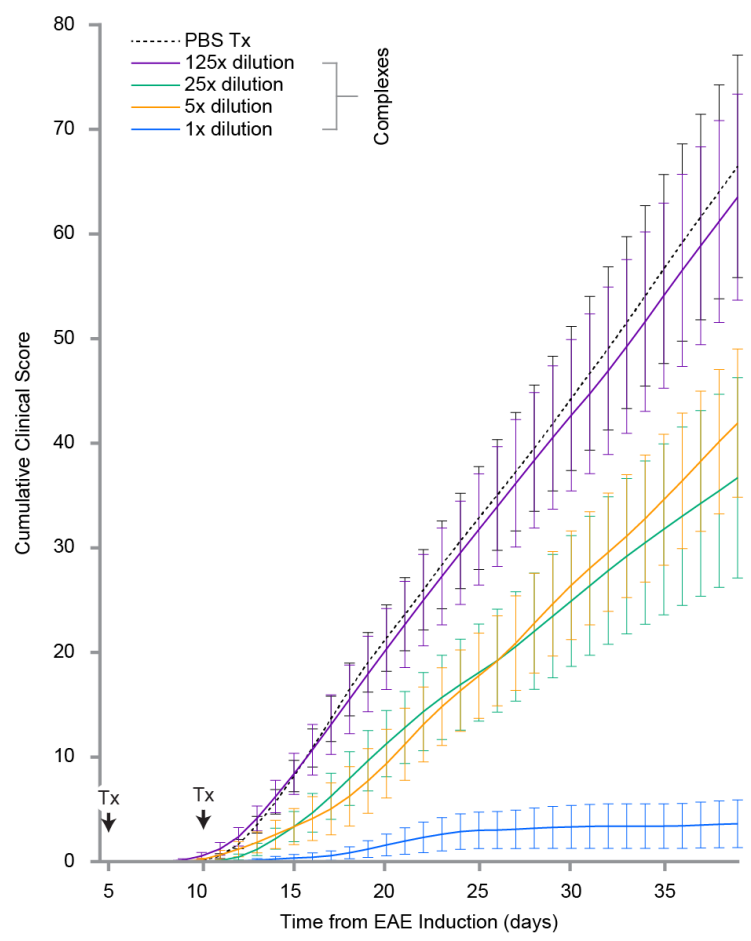

Fig. S2. Dose-dependent suppression of paralysis in EAE model of MS. EAE was induced in mice as a model of autoimmunity on Day 0. MOG / GpG complexes were administered at 5-fold dilutions (1x, 5x, 25x, 125x) on Days 5 and 10 through a subcutaneous injection. Mice were then monitored for clinical score based on level of paralysis and body weight. Cumulative disease score corresponding to Fig. 3C.
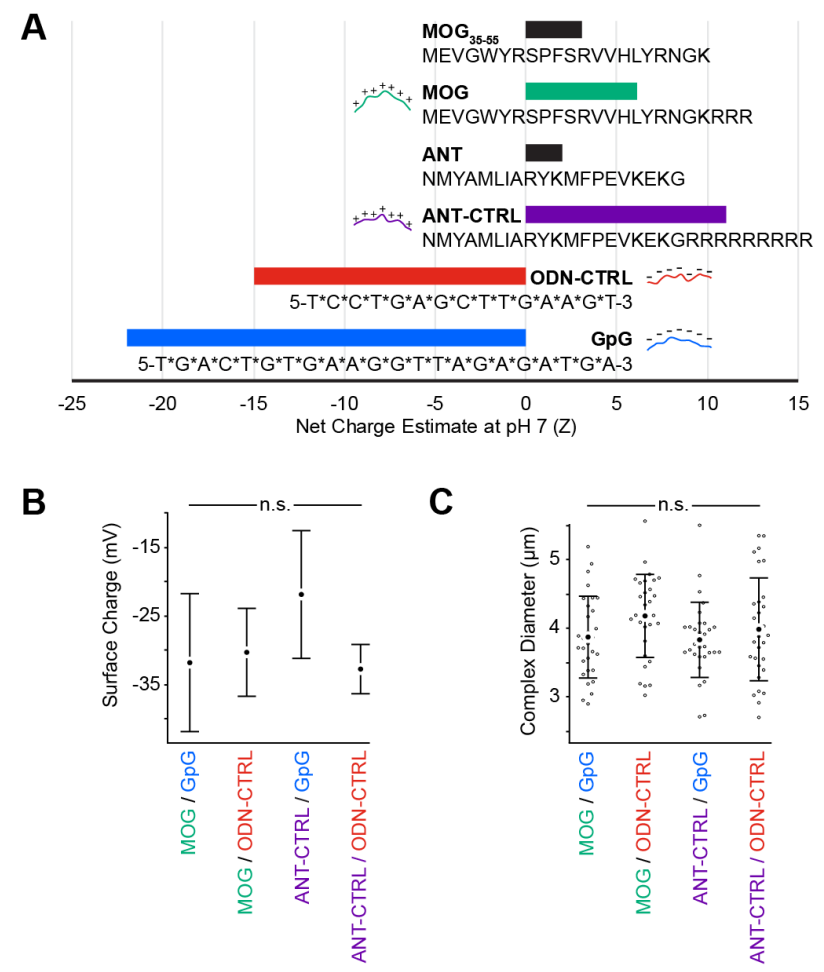

Fig. S3. Charge approximations for components, and surface charge and size of immune complexes. A) Peptide charge (Z) estimated by Innovagen Pepcalc and ODN charge estimated as -1 per nucleic acid. Complex microcapsule characterization for B) surface charge and C) microcapsule diameter. MOG complexes synthesized with GpG or ODN-CTRL were 3.88 \pm 0.56 and $4.18 \pm 0.61 \mu \mathrm{m}$ in diameter, respectively. ANTCTRL complexes synthesized with GpG or ODN-CTRL were 3.84 \pm 0.55 and 3.99 $\pm 0.75 \mu \mathrm{m}$ in diameter, respectively. 

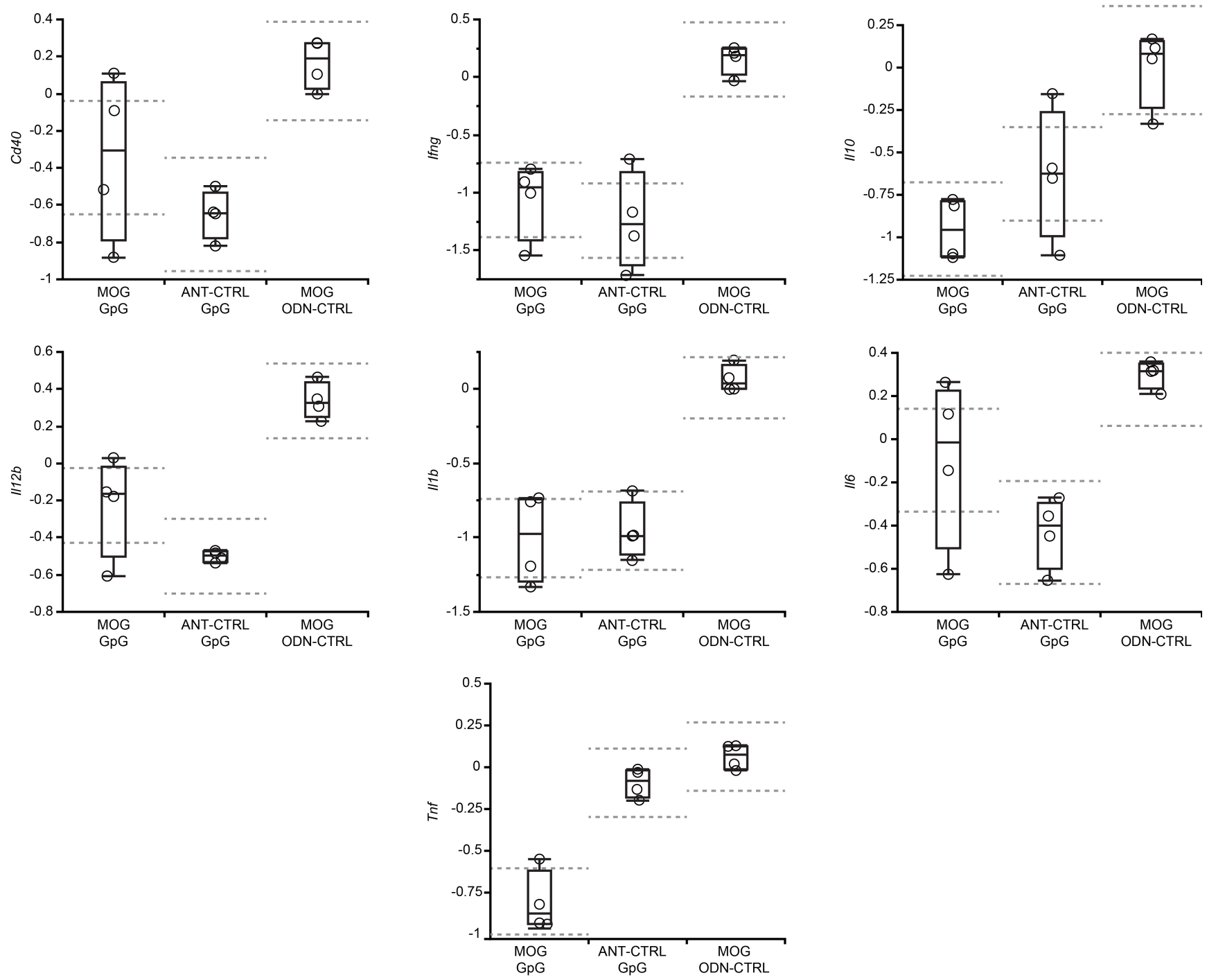

Fig. S4. GpG in complexes suppresses inflammatory gene expression. Gene expression in DCs isolated from spleens of C57BL/6 mice were analyzed following stimulation with $\mathrm{CpG}$ and treatment with the complexes formulations. Box plots match the unsupervised hierarchical clustering in Fig. 5B. Dashed lines in box plots represent 95\% confidence intervals. Data is represented as a log2 fold change on the y-axis. Each culture replicate (a data point, $n=4$ per condition) is an aggregation of $n=7$ technical treatment replicates.

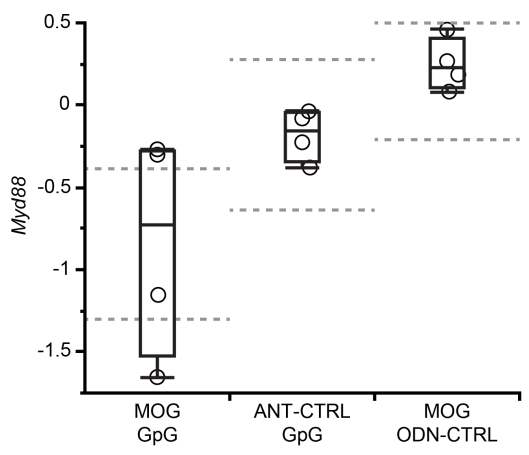

Fig. S5. GpG in complexes inhibits upstream Myd88 gene expression. Gene expression in DCs isolated from spleens of C57BL/6 mice were analyzed following stimulation with $\mathrm{CpG}$ and treatment with complexes. Box plots match the unsupervised hierarchical clustering in Fig. 5B. Dashed lines in box plots represent $95 \%$ confidence intervals. Data is represented as a log2 fold change on the y-axis. Each culture replicate (a data point, $n=4$ per condition) is an aggregation of $n=7$ technical treatment replicates. 

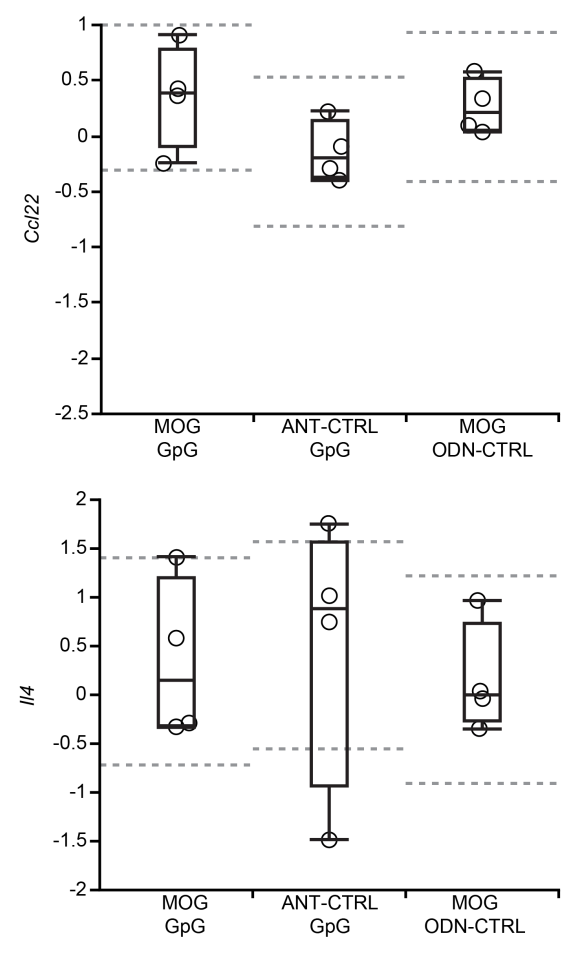

Fig. S6. GpG in complexes did not impact Ccl22 and II4 gene expression. Gene expression in DCs isolated from spleens of C57BL/6 mice were analyzed following stimulation with $\mathrm{CpG}$ and treatment with complexes. Dashed lines in box plots represent $95 \%$ confidence intervals. Data is represented as a $\log 2$ fold change on the $y$-axis. Each culture replicate (a data point, $n=4$ per condition) is an aggregation of $n=7$ technical treatment replicates. 

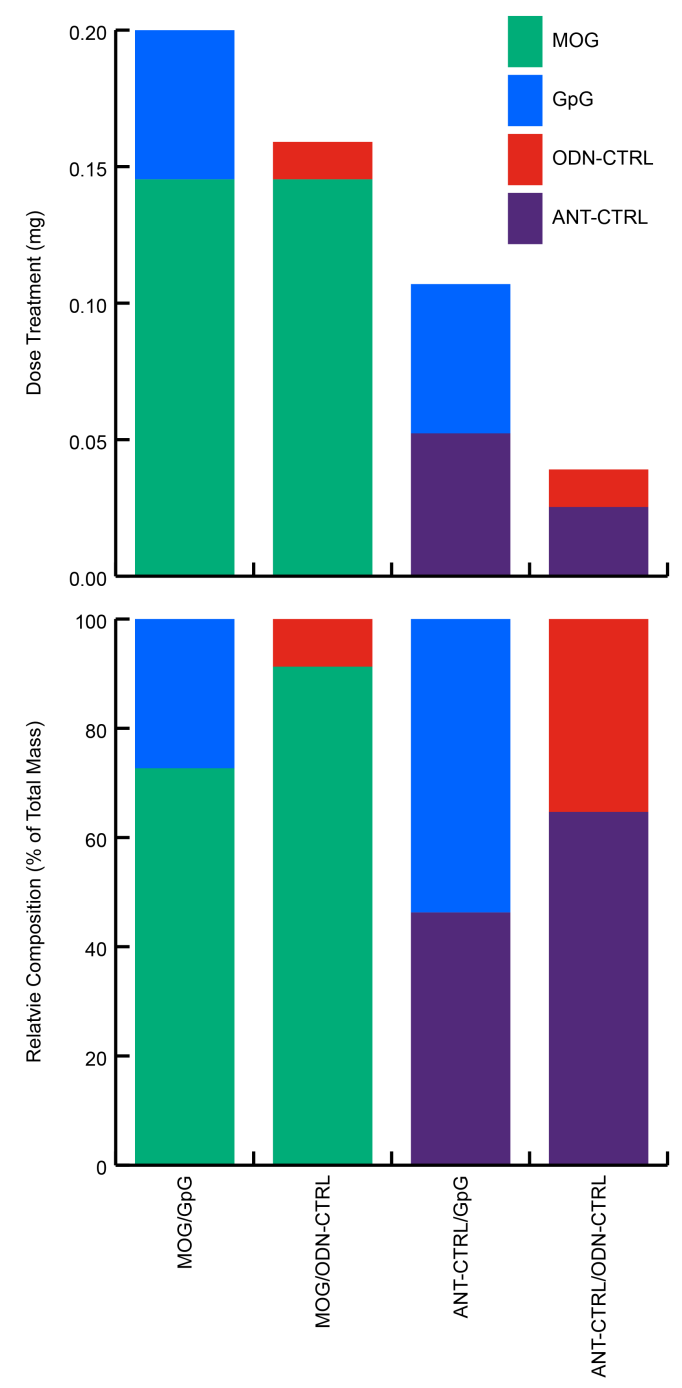

Fig. S7. Dose matching to isolate the role of each component. To isolate the role of MOG in complexes (Fig. 5), MOG was dose-matched between MOG/GpG and MOG/ODN-CTRL complex administrations. To isolate the role of GpG in complexes, GpG was dose-matched between MOG/GpG and ANT-CTRL/GpG complex administrations. ANT-CTRL/ODN-CTRL was dose-matched to the ODN-CTRL in the MOG/ODN-CTRL complexes. Top panel illustrates total mass of each component per dose and the bottom panel illustrates the relative dose in each treatment. 
A

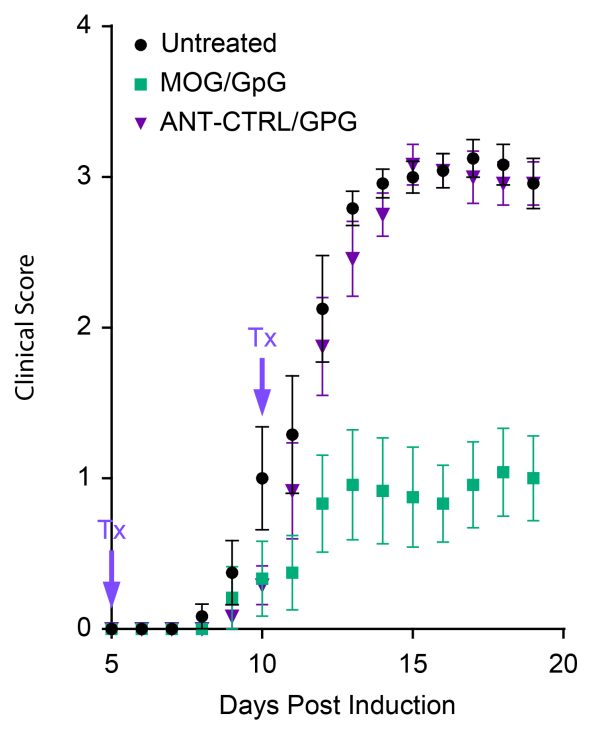

B

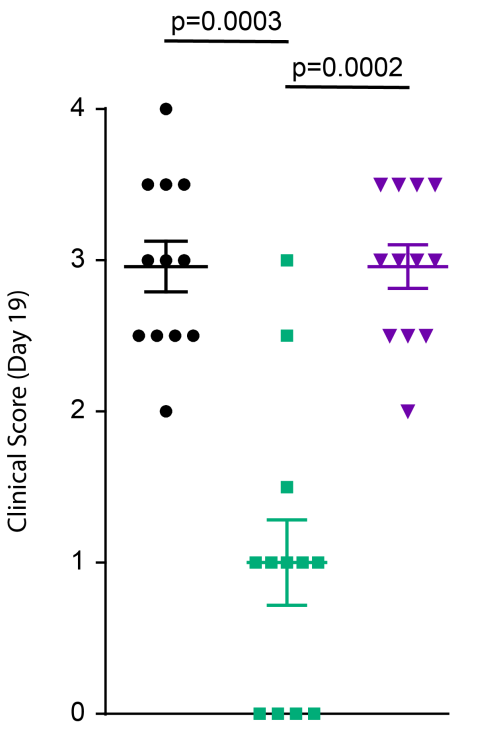

Fig. S8. Treatments require myelin self-antigen to suppress autoimmune driven paralysis. Similar to data in Fig. 5, complexes were synthesized to contain MOG/GpG or ANT-CTRL/GpG. A) EAE was induced on Day 0 with a 2x dose of PTX compared to data in Fig. 5 and complexes treatments (Tx) were administered on Days 5 and 10 through a subcutaneous injection. Clinical score based on the level of paralysis was tracked over time for the treatment groups. B) Clinical score on Day 19 is compared using a Wilcoxon test. $\mathrm{n}=12$ for all in vivo groups.
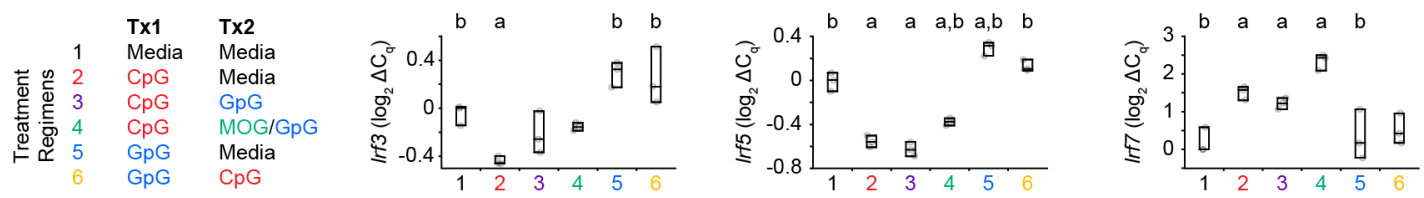

Fig. S9. GpG restrains CpG agonist function in IFN regulatory genes in vitro. Gene expression in DCs isolated from spleens of C57BL/6 mice were analyzed following treatment regimens in Fig. 6A. Box plots correspond to the heatmap in Fig. 6B. Data is centered on media control group with $\mathrm{y}$-axis indicated the log2 fold change. Statistical significance indicates an adjusted p<0.05 compared to media control (a) and CpG control (b) following a Tukey-Kramer multiple comparisons test. 